\title{
CONSIDERING TIME IN ORTHOPHOTOGRAPHY PRODUCTION: FROM A GENERAL WORKFLOW TO A SHORTENED WORKFLOW FOR A FASTER DISASTER RESPONSE
}

\author{
G. Lucas ${ }^{a, b, *}$ \\ ${ }^{a}$ Research Institute of Remote Sensing and Rural Development, Károly Róbert College, Gyöngyös, Hungary - \\ gregory.luc4s@gmail.com \\ ${ }^{\mathrm{b}}$ Doctoral School of Military Engineering, National University of Public Service, Budapest, Hungary
}

Commission IV, WG IV/7

KEY WORDS: Disaster Response, Orthophoto Production, Aerial Surveying, Data Acquisition, Post-processing, Shortened Workflow, Direct Geo-referencing, Reference Time Data.

\begin{abstract}
:
This article overall deals with production time with orthophoto imagery with medium size digital frame camera. The workflow examination follows two main parts: data acquisition and post-processing. The objectives of the research are fourfold: $1 /$ gathering time references for the most important steps of orthophoto production (it turned out that literature is missing on this topic); these figures are used later for total production time estimation; 2 / identifying levers for reducing orthophoto production time; 3 / building a simplified production workflow for emergency response: less exigent with accuracy and faster; and compare it to a classical workflow; 4/ providing methodical elements for the estimation of production time with a custom project.

In the data acquisition part a comprehensive review lists and describes all the factors that may affect the acquisition efficiency. Using a simulation with different variables (average line length, time of the turns, flight speed) their effect on acquisition efficiency is quantitatively examined.

Regarding post-processing, the time references figures were collected from the processing of a 1000 frames case study with $15 \mathrm{~cm}$ GSD covering a rectangular area of $447 \mathrm{~km}^{2}$; the time required to achieve each step during the production is written down. When several technical options are possible, each one is tested and time documented so as all alternatives are available. Based on a technical choice with the workflow and using the compiled time reference of the elementary steps, a total time is calculated for the post-processing of the 1000 frames. Two scenarios are compared as regards to time and accuracy. The first one follows the "normal" practices, comprising triangulation, orthorectification and advanced mosaicking methods (feature detection, seam line editing and seam applicator); the second is simplified and make compromise over positional accuracy (using direct geo-referencing) and seamlines preparation in order to achieve orthophoto production faster. The shortened workflow reduces the production time by more than three whereas the positional error increases from 1 GSD to 1.5 GSD. The examination of time allocation through the production process shows that it is worth sparing time in the post-processing phase.
\end{abstract}

\section{INTRODUCTION}

Natural or industrial disasters require immediate response to save lives and properties, to survey damages, to identify open access roads, etc. So the approximate estimation of the time necessary to produce orthophotography is an important issue for companies or scientists dealing with imagery support so as they can position themselves in the response phase. Another challenge is the ability to shorten the production time (and respectively adapt the production workflow) to meet the specific requirements of disaster response. This article copes with these two issues. All time references are established for a medium size digital frame camera (60 MP). Medium format digital camera offers the best flexibility nowadays with imagery for rapid response imaging. (Grenzdörffer, 2008)

Whatever part is considered: data acquisition and postprocessing parts, no detailed quantitative information is available in the scientific literature about production time. The only exception stands for systems performing real time orthophoto production. With these ones individual orthophotos are available immediately after the flight. (Applanix, 2009; Ip et al., 2002). The literature focuses on the description of workflows (direct georeferencing, Integrated Sensor Orientation
(ISO)), positional accuracy assessment and advanced uses of imagery data. Several papers mention the gain of time associated with direct geo-referencing (Rizaldy and Firdaus, 2012; Skaloud, 2007) without providing quantitative information.

Facing this miss, different post-processing tests were done with a test dataset and the time for data processing was collected. In the case of data acquisition, where many variables should be considered and interact, simulation was used to quantify several factors effect on acquisition efficiency. The factors affecting production time are listed (flight altitude and GSD, technical choice during processing, hardware equipment, etc.) and some of them are identified as possible lever to reduce production time. Many workflows are possible leading to orthophoto products varying in quality (radiometry, seam lines, accuracy). When several processing options are possible, each one is tested and time documented so as all alternatives can be considered for building workflows. In a following part, starting from a classical orthophoto production workflow and analysing which parts are over consuming time in comparison of their advantages we design a processing workflow adapted to disaster response (shorter in time). Next, the accuracy of the dataset obtained with the shortened workflow is compared to the

\footnotetext{
* Corresponding author
} 
accuracy obtained with the classical workflow and conclusions are given. In a last part, element of methodology are given to be able to extrapolate time references data to projects of any size.

\section{REVIEW OF THE FACTORS AFFECTING ACQUISITION EFFICIENCY}

\subsection{Definition of acquisition efficiency}

Acquisition efficiency with a digital camera can be defined as the average area surveyed per unit of time (for a given GSD). Another acquisition efficiency approach considers the average number of frames prepared per unit of time: a frame rate. Frame rate should always be employed with precaution as the multiplication of frame number other an area does not mean the acquisition is efficient (too much overlay is counterproductive (in post-processing)).

2.2. GSD/flight altitude pair and potential effects on acquisition and post-processing time.

$\mathrm{GSD}^{1}$ increases proportionally with flight altitude.

$\mathrm{GSD}=\mathrm{AAG} / \mathrm{f} \times \mathrm{a}^{\prime}$

where $\quad$ AAG $=$ Altitude Above Ground $(\mathrm{m})$

$\mathrm{f}=$ focal length $(\mathrm{m})$

$\mathrm{a}^{\prime}=\mathrm{CCD}$ pixel size (micron)

As a consequence, the acquisition time for a given area decreases if the project is flown higher (providing the percentage of overlay between images on a line and between lines remains unchanged). AAG also impacts on frame rate. If AAG increases, the distance between two images centres also increases, consequently time between image increases and the frame rate get lower. In all strictness it makes sense to compare frame rate only between projects flown at the same AAG.

\subsection{Flight speed}

The increase of flight speed allows reaching a higher efficiency.

\subsection{Short turns, smooth turns, IP time}

Two different types of turn can be set in the flight management system (smooth turn or short turn) as well as a time for the Initialisation Point (IP) before image acquisition starts.

\subsection{Geometry of the survey area}

Geometry influences very much acquisition efficiency. For example acquisition over a rectangular $\mathrm{AOI}^{2}$ last shorter than acquisition over an irregular AOI with the same number of frames because in the second one more turns and sometimes parts without acquisition should be flown to join the next line.

\subsection{Overlay in the block}

Classical photogrammetry blocks require $60 \%$ overlay inside a line and $20 \%$ between lines. In the case aerial triangulation is not performed but replaced by direct referencing, it could be worth trying to reduce the overlay on line and between lines to $10-15 \%$ (even less if the system is equipped with gyro-stabilize mount). The acquisition time decrease in parallel with the

\footnotetext{
${ }^{1}$ Ground Sampling Distance

${ }^{2}$ Area Of Interest
}

overlay between lines as the number of lines decreases. The decrease of overlay inside line doesn't affect the time for data acquisition (as the aircraft fly at the same speed), but decrease the number of frame to post-process afterwards.

\subsection{Aerial photography equipment characteristics}

Acquisition time closely depends on equipment characteristics. The reference time data in this paper are stated for a medium format digital frame camera.

\begin{tabular}{ll}
\hline Equipment & Characteristics \\
\hline RCD30 camera & Medium format digital camera (60 \\
& MP). RGBN. Focal: 53 mm. Pixel \\
& size: 6 micron, $9000^{*} 6732$ pixels. \\
Cesna 206 skywagon & Flight speed: $185 \mathrm{~km} / \mathrm{h}$ \\
\hline
\end{tabular}

Table 1. Description aerial surveying equipment

\subsection{Identification of levers for shortening acquisition time}

Some levers are routinely used in aerial surveying (like the flight altitude, the flight speed, the frame overlay) and their effects directly visible during the flight planning phase (effect rather visible on the geographical coverage than on timing). Some others (operational) like the turns patterns or the time set for the IP capture are on operational side and are rarely considered. They depend mainly on the pilot capacities and aircraft characteristics.

\begin{tabular}{lll}
\hline Step & Lever & Action \\
\hline $\begin{array}{l}\text { Flight } \\
\text { planning }\end{array}$ & $\begin{array}{l}\text { Flight altitude } \\
\text { (GSD) }\end{array}$ & $\begin{array}{l}\text { Altitude as high as possible = } \\
\text { reduce frame number = shorten } \\
\text { acquisition time = shorten post- } \\
\text { processing time. } \\
\text { decrease to 10-15\% decreases } \\
\text { the frame number (to post- } \\
\text { process). } \\
\text { decrease to 10-15\% decreases } \\
\text { the number of lines and } \\
\text { decreases the number of frames } \\
\text { to post-process. } \\
\text { planned at the maximum } \\
\text { inside line }\end{array}$ \\
Frame overlay \\
between line
\end{tabular}

Table 2. Possible levers for shortening data acquisition

\section{QUANTIFYING ACQUISITION TIME SHORTENING}

\subsection{Flight speed and turn pattern}

Figure 1 below was established using the following method. The input data is an average line length series, from $0.5 \mathrm{~km}$ to $60 \mathrm{~km}^{3}$. For each length we calculated the corresponding online flight time (using the flight speed). The time for one turn was added to this value; this results in a total raw time for one line (a). Using project geometry characteristics we calculated how many frames are prepared on each line based on its length and a $60 \%$ overlay between frames.

\footnotetext{
${ }^{3} 60 \mathrm{~km}$ is a maximal length; it corresponds to a bit less than 20 minutes of flight which is the time when IMU start to
} become inaccurate. 
frame number $(b)=$ line length $/\left(\left(1-0.6^{4}\right) \times\left(6732^{5} \times 0.15^{6}\right)\right)$ (2)

Finally frame rate value is obtained by dividing the frame number (b) by the total time (a).

Two different flight speeds were tested: $185 \mathrm{~km} / \mathrm{h}$ and 205 $\mathrm{km} / \mathrm{h}$.

Two time for the turns are tested: 3 minutes (smooth turn) and 2,3 minutes (short turn and reduce IP).

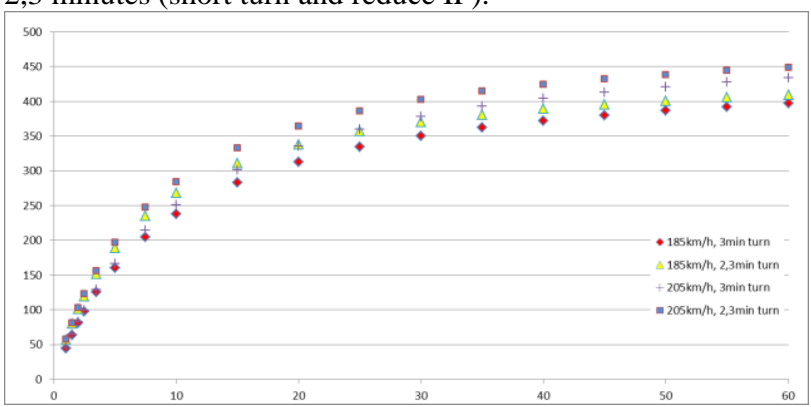

Figure 1. Effect of: average line length, speed and time of turn on frame rate.

Analysing figure 1, several conclusions can be formulated. The frame rate increases logarithmically with the average line length. The characteristics of the turns have more influence when the flight lines become shorter (effect of the line/turn ratio in favour of the turn). On the opposite the flight speed gain effect when lines become longer.

By combining flight speed and short turns it is possible to gain 50 frames per hour out of 300 , so an increase of efficiency of 16 percent. 50 frames cover an area of $49 \mathrm{~km}^{2}$ with $15 \mathrm{~cm}$ GSD and $15 \%-15 \%$ overlay.

\subsection{Frame overlay}

Decreasing frame overlay between lines from $20 \%$ to $10 \%$ increase efficiency accordingly. Decreasing frame overlay inside line from $60 \%$ to $15 \%$ does not affect acquisition time (as the aircraft coverage and speed remain unchanged) but reduces the number of frame to post-process by $35 \%$ which is significant.

\section{DATA PROCESSING: FACTORS TO CONSIDER AND METHODOLOGY TO FASTEN PRODUCTION}

\subsection{Number of bands and pixel depth}

Post-processing time logically increases with image size. The size of an image depends on the number of pixels, the number of bands (usually 1,3 or 4 ) and the depth of the pixels $(8,16$, 24 bits per band).

GSD has no effect on the processing time as the number of pixels per image remains constant whatever the GSD is. Consequently the number of frames and the size of an image are the best descriptors to dimension a project regarding postprocessing.

\subsection{Hardware and software description}

Post-processing time depends first of all on the computing capacities. This paragraph provides a brief technical description

\footnotetext{
${ }^{4}$ Due to the $60 \%$ overlay inside line

${ }^{5} \mathrm{CCD}$ width

${ }^{6} 15 \mathrm{~cm}$ GSD
}

about the hardware, equipment and software used in our orthoimagery production unit. By comparison with its own equipment, reader can estimate if his production capacity differs from the one given in the tables hereafter; and make adjustments to approach his production capacities.

\begin{tabular}{|c|c|c|}
\hline $\begin{array}{l}\text { Hardware } \\
\text { computing and } \\
\text { storage }\end{array}$ & \multicolumn{2}{|l|}{ Characteristics } \\
\hline $\begin{array}{l}\text { SGI Octane } \\
\text { workstation }\end{array}$ & \multirow{2}{*}{\multicolumn{2}{|c|}{$\begin{array}{l}16 \text { processors E5620 (Intel Xeon } 2.4 \\
\text { GHz). } \\
\text { "Z" main disk-array network transfer I/O } \\
\text { is } 2 \times 10 \mathrm{Gbps} \text { (this is the "star centre" in } \\
\text { network structure, and every node } \\
\text { connect to this with } 1 \text { Gbps net } \mathrm{I} / \mathrm{O} \text { ). }\end{array}$}} \\
\hline Network & & \\
\hline Software & Operations & Additional info. \\
\hline Framepro 1.3 & $\begin{array}{l}\text {.tiff generation, } \\
\text { project download }\end{array}$ & Free software \\
\hline $\begin{array}{l}\text { Leica } \\
\text { IPAS_TC_3.2 }\end{array}$ & $\begin{array}{l}\text { Trajectory } \\
\text { computation }\end{array}$ & \\
\hline $\begin{array}{l}\text { Leica } \\
\text { IPAS_CO_2.3 }\end{array}$ & $\begin{array}{l}\text { Image orientation } \\
\text { computation }\end{array}$ & \\
\hline $\begin{array}{l}\text { Trimble Inpho } 6.0 \\
\text { Application Master }\end{array}$ & Block build up & \\
\hline $\begin{array}{l}\text { Trimble Inpho } 6.0 \\
\text { MATCH-AT }\end{array}$ & Aerial triangulation & \\
\hline $\begin{array}{l}\text { Trimble Inpho } 6.0 \\
\text { OrthoMaster }\end{array}$ & Orthorectification & $\begin{array}{l}16 \text { process in } \\
\text { parallel }\end{array}$ \\
\hline $\begin{array}{l}\text { Trimble Inpho } \\
\text { OrthoVista }\end{array}$ & $\begin{array}{l}\text { Radiometric } \\
\text { enhancement, } \\
\text { mosaicking }\end{array}$ & $\begin{array}{l}16 \text { sub- } \\
\text { processed } \\
\text { allowed }\end{array}$ \\
\hline $\begin{array}{l}\text { Trimble Inpho } \\
\text { OrthoVistaSE }\end{array}$ & Seam editing & I \\
\hline
\end{tabular}

Table 3. Description of hardware and software

\subsection{Identification of levers for shortening production time}

This section introduces the technical means allowing the shortening of post-processing. Reader should notice that the selection of faster and/or simplified process (or eventually the skipping of processes) usually impacts on final orthophoto product quality (radiometry, seamlines or accuracy). Consequently levers should be employed carefully in order to both achieve time shortening and meet the technical requirements (radiometry, accuracy). Leverages are summarized in the table 4 .

\begin{tabular}{|c|c|c|}
\hline Step & Lever & Method \\
\hline $\begin{array}{l}\text { Tiff image } \\
\text { generation }\end{array}$ & $\begin{array}{l}\text { Nb. of channel }(\mathrm{RGB}, \\
\mathrm{RGBN}, \mathrm{RGB}+\mathrm{N}, \mathrm{N}) \\
\text { File format }(8 \text { or } 16 \\
\text { bits per band) }\end{array}$ & $\begin{array}{l}\text { Limited to the channel } \\
\text { used: example: RGB } \\
\text { and skip IR, } 8 \text { bits per } \\
\text { band }\end{array}$ \\
\hline $\begin{array}{l}\text { External } \\
\text { orientation }\end{array}$ & $\begin{array}{l}\text { Direct georeferencing } \\
\text { orthophoto } \\
\text { production or } \\
\text { workflow including } \\
\text { Aerial Triangulation } \\
\text { (AT) }\end{array}$ & $\begin{array}{l}\text { AT skipped } \\
\text { Use the values of PPA } \\
\text { and misalignment } \\
\text { calculated from a } \\
\text { calibration flight in EO } \\
\text { computation. }\end{array}$ \\
\hline $\begin{array}{l}\text { Orthorectif } \\
\text { ication }\end{array}$ & $\begin{array}{l}\text { Overlay between } \\
\text { images } \\
\text { Calculation method }\end{array}$ & $\begin{array}{l}\text { Reduced as much as } \\
\text { possible }(0 \%) \text {. } \\
\text { Chose a fast method }\end{array}$ \\
\hline Mosaicking & $\begin{array}{l}\text { Seam line calculation } \\
\text { Radiometry } \\
\text { improvement }\end{array}$ & $\begin{array}{l}\text { Using plain mosaic } \\
\text { None (no global } \\
\text { tilting) }\end{array}$ \\
\hline
\end{tabular}




\begin{tabular}{cc} 
Tiling & $\begin{array}{l}\text { Use tiling to avoid } \\
\text { processing empty areas }\end{array}$ \\
\hline
\end{tabular}

Table 4. Levers available for shortening workflow

\subsection{Measure of post-processing efficiency approached through time to process a given number of images (1000 images)}

Because of practical raisons, post-processing efficiency is not approached through the number of images processed per unit of time (example: orthorectification: 250 images per hours, mosaicking: 150 images per hour, etc.) but with the time required to process a given number of images. The first can easily be derived by calculation. The second offer the advantage to be additive, so as total production time can be calculated simply by adding elementary time values.

To set references time series, a reference (and significant) number of images have to be chosen. Small photogrammetry projects use several hundreds of images; big projects use several thousands. We chose an intermediary value of 1000 images for our case study for the compilation of processing time values.

\section{POST-PROCESSING TIME DATA COLLECTION}

Data collection was done with several repetitions in order to validate each of the figures compiled in table 3 . Special care was taken not to run processes in parallel in order not to generate bias. The processed 1000 images are 8 bits and 3 bands tiff images.

\begin{tabular}{|c|c|}
\hline Operation & Time \\
\hline \multirow[t]{3}{*}{ raw tiff calculation } & $3 \mathrm{~h} 04$ \\
\hline & $12.2 \mathrm{sec}$ per image \\
\hline & $11 \mathrm{sec}$ per image on $\mathrm{Z} /$ \\
\hline \multicolumn{2}{|l|}{ Orthorectification $^{7}$} \\
\hline Import in OrthoMaster project & $8 \min$ \\
\hline $\begin{array}{l}\text { Orthorectification } 150 \% \text { overlay, } \\
\text { cubic convolution, with complete set } \\
\text { of overview }\end{array}$ & $7 \mathrm{~h} 30$ \\
\hline $\begin{array}{l}\text { Orthorectification } 0 \% \text { overlay (fast } \\
\text { method, bilinear, no overview) }\end{array}$ & $1 \mathrm{~h} 36$ \\
\hline \multicolumn{2}{|l|}{ Feature detection } \\
\hline $\begin{array}{l}\text { Feature detection calculation Urban } \\
\text { on } \mathrm{Z} \text { (with tiling } 1200 \mathrm{~m} \text { ) }\end{array}$ & $10 \mathrm{~h} 48$ \\
\hline $\begin{array}{l}\text { Feature detection calculation Urban } \\
\text { on } \mathrm{Z} \text { (with big area) }\end{array}$ & $6 \mathrm{~h} 30$ \\
\hline $\begin{array}{l}\text { Feature detection rural algorithm on } \\
\text { G: (big area) }\end{array}$ & $3 \mathrm{~h} 41$ \\
\hline $\begin{array}{l}\text { Feature detection urban algorithm on } \\
\text { Y: with } 1200 \text { tiling }\end{array}$ & $9 \mathrm{~h} 36$ \\
\hline $\begin{array}{l}\text { Feature detection urban algorithm on } \\
\text { Y: with big aera (16 sub process } \\
\text { parallel) }\end{array}$ & $8 \mathrm{~h} 51$ \\
\hline Seam line editing & $\begin{array}{l}\text { will vary based on entropic } \\
\text { elements in the AOI. Consider } \\
5 \mathrm{~h} \text { for } 1000 \text { images. }\end{array}$ \\
\hline adaptive feathering (mixed) & $6 \mathrm{~h} 15$ \\
\hline \multicolumn{2}{|l|}{ Mosaicking } \\
\hline $\begin{array}{l}\text { Plain mosaic } 1200 \mathrm{~m} \times 1200 \mathrm{~m} \text { on } \\
\text { orthos with } 0 \% \text { overlay }\end{array}$ & $1 \mathrm{~h} 17$ \\
\hline
\end{tabular}

\footnotetext{
7 * Orthorectification process requires a digital elevation model. In our practice elevation data is collected meantime with imagery (with ALS 70) and DTM produced prior to its use in orthorectification. Intentionally we have not considered how DTM production time fit into the orthophoto production time but only concentrate on the orthophoto processes. To be exhaustive the DTM production time should be considered.
}

\begin{tabular}{|c|c|}
\hline Plain mosaic one big area & $4 \mathrm{~h} 13$ \\
\hline Plain mosaic $1200 \mathrm{~m} \times 1200 \mathrm{~m}$ tiles & 1h55 \\
\hline $\begin{array}{l}\text { Mozaic with seam applicator } \\
1200 \times 1200 \text { tiles }\end{array}$ & $1 \mathrm{~h}$ \\
\hline $\begin{array}{l}\text { Mozaic with seam applicator } \\
1200 \times 1200 \text { tiles on } \mathrm{Y} \text { (+adaptative } \\
\text { blending urban) }\end{array}$ & 1h14 \\
\hline $\begin{array}{l}\text { Mozaic with seam applicator } \\
1200 x 1200 \text { tiles on } \mathrm{Y} \text { (+adaptative } \\
\text { blending rural) }\end{array}$ & $3 \mathrm{~h} 08$ \\
\hline $\begin{array}{l}\text { Mozaic with adaptive feathering } \\
\text { urban } 1200 \times 1200 \text { tiles on Y }\end{array}$ & $6 \mathrm{~h} 50$ \\
\hline $\begin{array}{l}\text { Mozaic with adaptive feathering rural } \\
1200 \times 1200 \text { tiles on } Y\end{array}$ & $6 \mathrm{~h} 12$ \\
\hline
\end{tabular}

\section{PROPOSITION OF REFINED WORKFLOW FOR EMERGENCY MANAGEMENT SITUATION}

\subsection{Workflow shortening method}

First let's consider the fastest processing option for each step: Image absolute orientation

If the accuracy requirements do not go lower than 1.5 GSD, direct referencing method can profitably be applied instead of aerial triangulation. Qtaishat demonstrated that directgeoreferencing achieves sufficient accuracy in the case of medium to small sized projects with correct selection of GPS base station (Qtaishat et al., 2006). Rizaldy mentions both the accuracy and the fastness of direct referencing method (Rizaldy and Firdaus, 2012). Aerial triangulation is time consuming because it requires several operations $\left(\mathrm{APM}^{8}\right.$, field measurements of control points (it can be problematic in impacted zones) and triangulation). In direct geo-referencing accuracy is ensured by a calibration flight and misalignment calculation ( $\mathrm{PPA}^{9}$ and misalignment are used for external orientation calculation) (Mostafa, 2003). So calibration should be placed in the workflow.

\section{Orthorectification}

Time is spared with orthorectification choosing a fast calculation method, $0 \%$ overlay between tiles and bilinear resampling method.

\section{Mosaicking}

The plain mosaic option is used, this avoid two operations: the feature detection and the seam applicator or the feathering which is also time consuming. No manual editing or the seamlines is done. Tiling is applied to avoid image generation in empty areas.

\section{Radiometry}

No radiometric improvement is applied (with global tilting or radiometrix tool).

Last we try to imbricate steps and run parallel processes when it is possible ( $\mathrm{Li}$ and $\mathrm{Zhan}, 2005)$. As an example the calibration procedure is short enough to fit during project download and image generation (see figure 2)). Calibration results are necessary for the calculation of the external orientations of images (red arrow). Similarly trajectory calculation can be done during tiff image generation. All the other processes can only be applied successively. Time figures for the selected steps were picked from table 5 and Gantt charts built for a shortened and a normal workflow.

\footnotetext{
${ }^{8}$ Automatic Point Matching

${ }^{9}$ Principal Point of Alignment
} 


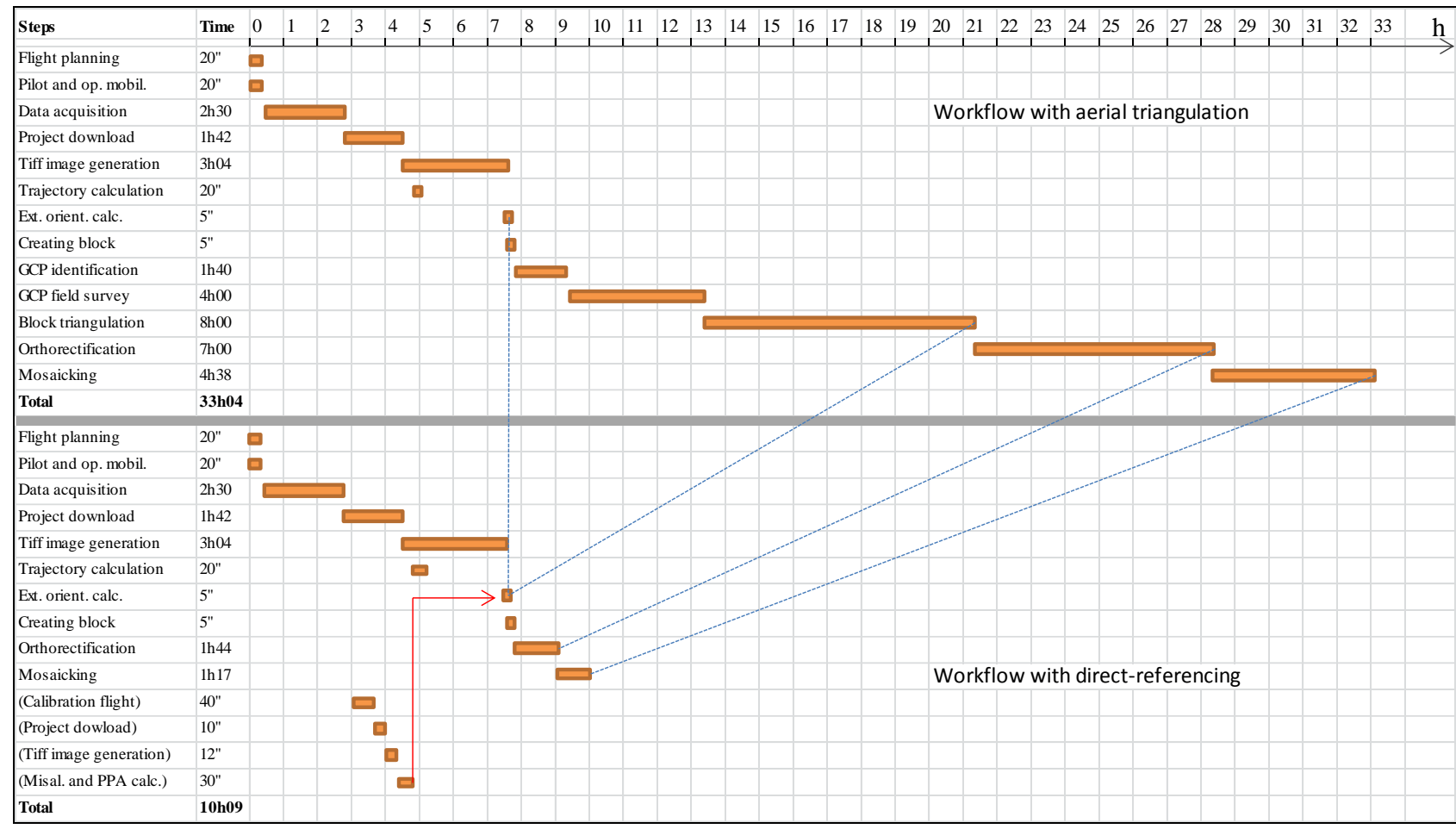

Figure 2. Gantt chart with comparison of normal and shortened workflows

\subsection{Refined workflow and associated processing time}

Figure 2 presents the two Gantt charts with the detail of time for every step and the total production time.

Most of the time is spared by replacing aerial triangulation by direct referencing plus a calibration flight (13h40). The calibration flight does not "cost" any time as it can be placed in parallel with another process ${ }^{10}$.

The comparison of the time required for the production of a 1000 images, 8 bites, 3 band project with a normal workflow (35 hours) and a shortened workflow (11h hours) shows that with judicious technical choices time can be reduced by more than three.

In the normal workflow, most of the time is required for postprocessing. In the fast workflow, acquisition represents a bit less than $50 \%$ of time use.

\subsection{Accuracy assessment of orthophotography produced with direct georeferencing}

Planimetric accuracy assessment was done with the measure of 15 control points with a $15 \mathrm{~cm}$ GSD dataset on the orthorectified images. It resulted with a RMSE of $28 \mathrm{~cm}$ in X and $15 \mathrm{~cm}$ in Y. Bibliography provide value of $27.4 \mathrm{~cm}$. (Mostafa and Hutton, 2010) Those values correspond with the 1.5 GSD generally cited in the literature for direct georeferencing (Mostafa, 2010). In comparison a RMSE (AT) of 0.5 GSD could be reached with aerial triangulation and sufficient number of control points in a classic photogrammetry workflow which represent a 1 GSD RMSE for the end product. (ASPRS, 2014).

\footnotetext{
${ }^{10}$ In practice a calibration flight can be systematically done after a shutter replacement or after system was mounted so as direct referencing can be done anytime without prior calibration.
}

\subsection{Generalisation of production time estimation to project of any size}

This paragraph provides a generalization method for the calculation of total production time for a project of any size (with the fast production workflow). Examples of calculations are presented for projects varying from 100 to 3000 frames. The different production steps are detailed. The average flight line length is calculated using: the size of image foot print (formula (1)), the percentage of overlays and a rectangular geometry of the block where length $=2 \times$ width. The overlay between frames was kept at $20 \%-60 \%$. Frame rate is determined using figure 1, and then net flight time is calculated. Download, tiff generation, orthorectification and mosaicking increase proportionally with the number of image processed. "8" procedure and flight planning have constant values.

\begin{tabular}{|c|c|c|c|c|c|c|}
\hline $\begin{array}{l}\text { Number of } \\
\text { frame }\end{array}$ & 100 & 250 & 500 & $\begin{array}{c}1000 \\
11\end{array}$ & 2000 & 3000 \\
\hline $\operatorname{Area}\left(\mathrm{km}^{2}\right)$ & 44 & 105 & 215 & 447 & 882 & 1309 \\
\hline $\begin{array}{l}\text { Av. line } \\
\text { length }(\mathbf{k m})\end{array}$ & 9,2 & 14.6 & 20.5 & 29 & 42 & 51 \\
\hline Nb. frame/h & 280 & 333 & 364 & 402 & 425 & 439 \\
\hline Planning & $0: 20$ & $0: 20$ & $0: 20$ & 0:20 & $0: 20$ & $0: 20$ \\
\hline $\begin{array}{l}2 \times \quad 8 " \\
\text { procedure }\end{array}$ & $0: 20$ & $0: 20$ & $0: 20$ & 0:20 & $0: 20$ & $0: 20$ \\
\hline Flight & $0: 21$ & $0: 45$ & $1: 22$ & $2: 29$ & $4: 42$ & $6: 50$ \\
\hline Download & $0: 10$ & $0: 25$ & $0: 51$ & $1: 42$ & $3: 24$ & $5: 06$ \\
\hline Tiff & $0: 15$ & $0: 45$ & $1: 31$ & 3:04 & $6: 02$ & 9:09 \\
\hline Orthorectif. & $0: 10$ & $0: 26$ & $0: 52$ & $1: 44$ & $3: 23$ & $5: 12$ \\
\hline Mosaicking & 0:06 & $0: 14$ & $0: 27$ & 1:17 & $1: 52$ & $3: 51$ \\
\hline Total time & $1: 42$ & $3: 15$ & $5: 43$ & $10: 56$ & 20:03 & $30: 48$ \\
\hline
\end{tabular}

Table 6. Calculation of total time for different sizes of projects

\footnotetext{
${ }^{11}$ Reference time
} 
Those figures also help to define a strategy if important areas should be flown (more than 1000 images). It appears it is worth flying the highest priority area, land and exchange memories in order to have orthophoto of half the coverage on the same day (after 10-12 hours) rather than flying as much as possible and get all the data in one time but after 24 hours.

\section{DISCUSSION}

\subsection{Data acquisition}

The application of the method developed in this work is simple and adapted to the prevision of acquisition time for projects with regular geometry. Considering projects with irregular geometries, the most reliable approach consists of running a flight simulation (with FCMS flight simulator) with the flight plan to test and to note the time spent between the start of the first line and the completion of the last line. Then the time of the two " 8 " procedures and taxi time should be added. The flight plan should be prepared with automatic search of the nearest line so as the simulator can automatically complete the flight in accelerated mode. Predictive modelling with multivariate modelling could also be an alternative but seems more complicated compared to the simulation option (already existing).

\subsection{Post-processing}

This study demonstrated that a shortened workflow allows fast data production: $447 \mathrm{~km}^{2}$ with $15 \mathrm{~cm}$ GSD in approximately 11 hours. For disaster response, immediate or few hours production time would be preferable. The result achieved so far - below a half day of production with a standard medium format imagery system - is on one hand modest in comparison of solutions developed for ultra-fast mapping (georeferenced orthophotos produced while still-in-the-air for military response; and fast mosaic production within several hours post flight) but on the other hand fast enough to meet responders needs (the workflow has the advantage to provide imagery coverage it the same day). Additionally it should be noted that $447 \mathrm{~km}^{2}$ is already a quite large area. The table 6 shows that production time fall under few hours for area smaller than 215 $\mathrm{km}^{2}$. Additional shortening (1h17) can be gained with ending the workflow after the orthorectification. Another shortening was mentioned but not yet attempted; it consists in reducing the overlay directly at the planning stage in order to achieve 10$15 \%$ overlay between frames. Such a practice could cause gap problems, so it should be tested first; secondly the data do not allows normal aerial triangulation, neither stereo measurement. In comparison, large aircrafts must fly high to capture images (or satellites) and the cloud deck often impedes the image capture. Large format cameras can hardly collect efficiently data for small and irregular areas.

We demonstrated that quite some time can be spared on the post-processing. Tightening data acquisition is possible but not so efficient in time shortening. So we recommend to perform data acquisition normally (in order to have qualitative raw data) and produce imagery coverage in two steps. The first production issues rush data with the shortened workflow; then with a standard workflow, coverage with optimal planimetric accuracy, radiometry and seamlines edition can be issued.

\subsection{Further work}

Testing if the division and processing of a project in parts offers a significant advantage regarding availability of data is a topic of interest. In this paper, each step of the post-processing handles $100 \%$ of the imagery and the whole coverage is available only at the end of the processing. The alternative we consider (which seems particularly interesting in the case of larger areas) would make possible to provide the coverage by part along the production cycle; this would constitute a significant advantage for the disaster management authorities as they could get the support of imagery earlier in the response phase (within several hours in this case). Different segmented workflows (with varying number of data restitutions and varying division of the AOI) could be tested.

\subsection{Interest to other parties}

More generally, time estimation is relevant for many parties dealing with orthophoto production for a better monitoring/management of projects, fleet, production and last but not least costs. Nowadays orthophoto production is moving towards the production of higher amount of data for lowers cost in a very competitive market. The correct and precise appreciation of production time (and costs) offers a competitive advantage as quotations can be calculated closest to the reality and projects better managed.

Secondly, direct georeferencing technique can be employed for projects where positional accuracy is superior or equal to 1.5 GSD to spare time and costs in combination with classic radiometry enhancement, orthorectification and seamlines edition technics.

\section{CONCLUSION}

The model developed in the data acquisition part showed that the frame rate increases logarithmically with the average line length. The model helped us to simulate projects with different flight lengths and to better measure the effects of factors like the flight speed and turn pattern on acquisition efficiency. The frame rate information read on the curve is used to predict the acquisition time for project with regular geometry. The model is not adapted to predict the acquisition time of projects with irregular or linear geometry. In this case it is better to prepare a flight plan and simulate the flight in a proprietary simulator. In the post-processing part, the comparison of the two workflows demonstrated that direct georeferencing is adapted to rapid response both for the shake of time and accuracy. The shortened workflow reduces the production time by more than three whereas the increase of the positional error remains reasonable (reaching around 1.5 GSD).

The examination of the time allocation, the levers for shortening and the consequences on data quality shows it is worth sparing time in the post-processing phase. The best production strategy would consist of making the appropriate data acquisition and then making two different productions, a first one with the shortest workflow for faster disaster response (rush data) and a second one lasting longer and following a classical workflow with optimal positional accuracy, enhanced radiometry and adequate work on seam lines.

The last lever usable to shorten production time is the reduction of the overlay between the frames at the planning stage. This option has the advantage to reduce the number of frames to post-process and also increase geographic coverage per time unit (and acquisition efficiency). Nevertheless it has the disadvantages to increase the risk of gap in the coverage, and 
the limited overlay does not allow aerial triangulation or stereo measurement. This option is advantageous if just directgeoreferencing and just orthoimagery product are foreseen. This option was considered theoretically but not implemented yet in practice. In theory $10 \%$ time can be spared with the flight time (if overlay between line is decreased from $20 \%$ to $10 \%$ ) and $50 \%$ time spared with post-processing (if overlay inside line is decreased from $60 \%$ to $10 \%$ ) which really is significant.

\section{REFERENCES}

Applanix, 2009. Applanix DSS ${ }^{\mathrm{TM}}$ RapidOrtho ${ }^{\mathrm{TM}}$ Solution. reference time data. http://applanix.com/media/downloads/ products/brochures/DSS_RapidOrtho_Overview.pdf (accessed: 01 Jul. 2015)

ASPRS, 2014. Positional Accuracy Standards for Digital Geospatial Data. Reprot. EDITION 1, Version 1.0, Nov. 2014.

Grenzdörffer, G. J., 2008. Medium format digital cameras - A EuroSDR project. In: The International Archives of the Photogrammetry, Remote Sensing and Spatial Information Sciences, Beijing, China, Vol. XXXVII, Part B1, pp. 10431050.

GIScafé, 2009. Applanix Introduces DSS RapidOrthoTM 2.0 for High-Speed Orthophoto Production.

http://www10.giscafe.com/nbc/articles/1/735525/ApplanixIntroduces-DSS-RapidOrthoTM-2.0-High-Speed-OrthophotoProduction (accessed 01 july 2015)

Ip, A. W. L., Mostafa , M. M. R., El-Sheimy, N., 2004. Fast orthophoto production using the digital sensor system. Proceedings of Map India 2004, New Delhi, India, January 2831, pp 79-84

Li, H., Zhan, D., 2005. Workflow Timed Critical Path Optimization. Nature and Science, 3(2), 2005

Mostafa, M. R., 2003. Design and Performance of the DSS. Proceedings, 49th Photogrammetric Week, Stuttgart, Germany, September 1-5.

Mostafa, M.M.R., Hutton J, 2010. Theoretical ground accuracy analysis derived from today's airborne digital frame cameras and direct georeferencing. ASPRS 2010 Annual Conference, San Diego, California, April 26-30 2010.

Qtaishat, K. S., Smith, M. J., Park, D. W. G., 2006. Assessing the performance of different direct-georeferencing strategies. ASPRS 2006 Annual Conference, Reno, Nevada. May 1-5, 2006.

Rizaldy, A., Firdaus, W., 2012. Direct georeferencong: a new standard in photogrammetry for high accuracy mapping, Melbourne, Australia. In: International Archives of the Photogrammetry, Remote Sensing and Spatial Information Sciences, Volume XXXIX, Part B1, pp. 5-9.

Skaloud, Jan., 2007. Reliability of Direct Georeferencing Beyond the Achilles' Heel of Modern Airborne Mapping. Photogrammetric Week 2007, pp. 227-241

\section{ACKNOWLEDGEMENTS}

This research was supported by the TÁMOP programme to the Károly Róbert College (TÁMOP-4.2.2.D-15/1/ KONV-20150010). 